\title{
Chamameceros exóticos
}

\author{
Representaciones de un género musical \\ a través de un repertorio de sonoridades \\ Emanuel Obregón \\ [UNNE-UNL]
}

\begin{abstract}
RESUMen Este artículo presenta un análisis sonoro-discursivo del segmento "Chamameceros Exóticos" correspondiente al ciclo radiofónico Los Chamameceros, producido y conducido por Antonio Tarragó Ros, transmitido por Radio Nacional Rosario entre 2007-2019. El objetivo del análisis es reflexionar cómo se manifiestan las representaciones sociales (Moscovici, 1985; Jodelet, 2002) y de qué manera configuran un paradigma estético musical (García, 2010) del "Chamamé exótico". Se tipifica un conjunto de atributos (características técnicas e instrumentales, organización del material musical, interpretación musical, etc.) para establecer un repertorio de sonoridades que permite identificar al "Chamamé exótico" en contraposición al "Chamamé canónico". La exploración conjunta del entramado sociodiscursivo y del agrupamiento selectivo de obras musicales que Tarragó Ros - en tanto representante destacado del género musical, cuyo estatus social y roles asumidos son reconocidos en un amplio campo cultural (Merriam, 1979) — realiza a través de un medio de radiodifusión de alcance nacional, nos permite describir un mecanismo de "autoexotismo" (Izquierdo-König, 2016) que revela una apertura del Chamamé hacia otros espacios simbólicos de reconocimiento y aceptación.
\end{abstract}

Palabras clave: chamamé, autoexotismo . representaciones · paradigma estético
Summary This article presents a sound-discursive analysis of the "Chamameceros Exóticos" segment corresponding to the Los Chamameceros radio show, produced by Antonio Tarragó Ros, broadcast by Radio Nacional Rosario between 2007-2019. The objective of the analysis is to reflect on how social representations are manifested (Moscovici, 1985; Jodelet, 2002) and in what way they configure a musical aesthetic paradigm (García, 2010) of the "exotic Chamamé». A set of attributes is typified (technical and instrumental characteristics, organization of the musical material, musical performance, etc.) to establish a repertoire of sounds that allows identifying the "exotic Chamamé» as opposed to the "canonical Chamamé". The joint exploration of the socio-discursive framework and the selective grouping of musical pieces that Tarragó Ros - as an outstanding representative of the musical genre, whose social status and assumed roles are recognized in a wide cultural field (Merriam, 1979) — carries out through a medium of broadcasting of national scope, it allows us to describe a mechanism of "autoexoticism" (Izquierdo-König, 2016) that reveals an opening of the Chamamé towards other symbolic spaces of recognition and acceptance.

Keywords: Electroacoustic Music . Nationalism · Cosompolitanism · Córdoba · Soundscape 


\section{INTRODUCCIón}

El músico Antonio Tarragó Ros ${ }^{1}$ produjo y condujo —entre 2007 y 2019- un ciclo radial titulado Los Chamameceros, inicialmente emitido por Radio Nacional Rosario, repetido por otras decenas de emisoras del país y, recientemente, retransmitido a través del portal web de Radio Nacional. En distintas oportunidades, esta producción radiofónica fue ternada y galardonada con el premio Martín Fierro Federal. ${ }^{2}$

Durante este ciclo radial Tarragó Ros produjo un segmento de programas especiales a los que tituló Exóticos. En palabras suyas, se trataba de "un programa raro, lo que podría llamarse "chamameceros exóticos". O sea, chamameceros que no lo son habitualmente» (Los Chamameceros $N^{\circ}$ g: Exóticos $N^{\circ}$ I). Este segmento de Chamameceros exóticos ponía al aire la música de una gran variedad de artistas nacionales e internacionales de diversa índole y con diferentes trayectorias, todos ellos interpretando Chamamé. La locución de Tarragó Ros enmarcaba discursivamente cada obra reproducida presentando a los intérpretes, mencionando a los compositores, refiriendo aspectos específicos de la producción musical (denominados «arreglos» $\mathrm{o}$ «versiones»), o bien aludiendo a rasgos sonoros de las obras reproducidas (indicando $v$.gr., características de una orquestación en particular, descripción de un estilo interpretativo, o bien, características tímbricas de las voces, así como otras impresiones personales respecto de arreglos vocales e instrumentales, etc.).

\footnotetext{
1 Antonio Tarragó Ros es un músico argentino de Chamamé, nacido en 1974 en Curuzu Cuatiá (Corrientes). En su trayectoria artística produjo y publicó decenas de álbumes en los que difundió su obra como autor y compositor. Sus obras de mayor difusión y reconocimiento han sido: Chamamecero (1981), Pueblero de allá ité (1983) y Curuzucuateño (1997), entre otras. En el presente trabajo nos basamos en su labor en radiofonía, cuya producción del ciclo radial llamado Los Chamameceros ha sido galardonado con el Martín Fierro Federal en 2008, además de haber sido ternado en otras oportunidades (asimismo, también ha recibido otros numerosos reconocimientos como Premio Estrella de Mar en 1988; Premio Konex en 1985 y 1995; Plaqueta de Oro ACE en 1993; Camín Cosquín de Oro en 1991 y Cóndor de Oro en 2007).

2 La dirección general de este programa estaba a cargo de Antonio Tarragó Ros. En la producción general: Irupé Tarragó Ros, María Ángela Lescano, Juan cruz Guillen, Carlos Serial, Cristina Hun. En la grabación y edición: Trabuco González, Hugo Mena, Miguel Angel Teti. El archivo completo de todas las emisiones de Los Chamameceros (270 programas en total) me fue entregado por Antonio Tarragó Ros especialmente para la realización de este artículo.
} 
De tal modo, se fue conformando un repertorio de exóticos con artistas como, entre otros: Mercedes Sosa, Lolita Torres, Suna Rocha, Eduardo Falú, Tamara Castro, Ariel Ramírez, Alberto Cortéz, Ricard Miralles, Bebu Silvetti, Bernhard von Der Goltz, Jorge Fandermole, Liliana Herrero, Yamila Cafrune, Los Cantores de Quilla Huasi, Chaqueño Palavecino, Horacio Guaraní, Los Chalchaleros, León Gieco, Miguel Cantilo y Ramón Ayala. Tarragó Ros se incluye a sí mismo en este repertorio, en el que participa desempeñando roles de coproductor, intérprete y arreglador.

La escucha de esta variada nómina de artistas, así como la multiplicidad de rasgos sonoros (entre otros múltiples aspectos que se conjugan en este repertorio), nos conduce a interrogarnos: ¿qué rasgos diferencian lo exótico de lo canónico en la práctica musical del Chamamé? Asimismo, ¿qué factores determinan el estar «dentro» o «fuera» del género? ¿Cómo suena un chamamé exótico? En particular, también surgen interrogantes respecto de los juicios estéticos presentes en el discurso de Tarragó Ros sobre las obras reproducidas en estos programas radiales; específicamente, aquellos que definen a los artistas y las obras que son «externos-al-chamamé» (Los Chamameceros $N^{\circ} 24$ : Exóticos $N^{\circ} 2$ ). ${ }^{3}$

Frente a esta problemática nos proponemos reflexionar sobre el modo en que las representaciones sociales manifiestas en el discurso de Tarragó Ros, entendidas como percepciones compartidas socialmente (Moscovici, 1985, Jodelet, 2002), configuran un "paradigma estético» (García, 20Io) del Chamamé canónico a la vez que también configuran un «autoexotismo» (Izquierdo-König, 2016). ${ }^{4}$

De manera preliminar, realizamos una aproximación a cómo estas dinámicas de inclusión y exclusión posicionan al Chamamé frente al

3 Incorporo operativamente la noción de repertorio en el sentido definido por Thibaudeau (2007: 24), no solo como un cuerpo de obras seleccionadas para ser interpretadas (que, a su vez, reciben modificaciones y cambios en cada interpretación) sino también como una convergencia de "datos objetivos (críticas, artículos publicados sobre la obra desde su creación) y subjetivos (recuerdos, leyendas y mitos forjados a lo largo de los años, etc.)".

4 Adoptaré la noción de representaciones sociales por su «valor heurístico (...) para comprender las interacciones sociales" (Abric, 1994) y habilitar la posibilidad de caracterizar el posicionamiento y pertenencia social de los sujetos en relación con su comunidad (Jodelet, 1988). 
campo artístico y cultural de la música popular argentina y las discursividades del nacionalismo cultural (Díaz, 2009).

\section{TARragó ROS, REPRESENTANTE DEL CHAMAMÉ}

Desde una perspectiva etnomusicológica, asumimos que Antonio Tarragó Ros es un sujeto socialmente diferenciado dentro del campo cultural y artístico de la música popular argentina, en tanto desempeña roles específicos y posee un estatus social, los cuales se encuentran determinados por el reconocimiento público (Merriam, 1979). En ese sentido, no solo es un sujeto reconocido por su rol como músico (intérprete y compositor), sino también como artista (productor artístico, productor y coproductor musical). A ello se suma su rol de comunicador social (conductor y productor radiofónico) en un medio de radiodifusión de alcance nacional. Por dicha labor como comunicador social también obtuvo reconocimiento público mediante el Premio Martín Fierro Federal, un tipo de galardón reservado a producciones televisivas y radiales de alcance nacional, otorgado por la Asociación de Periodistas de la Televisión y la Radiofonía Argentinas (APTRA). Este Martín Fierro Federal es una «variante», originada en I99I, para premiar especialmente a las producciones televisivas y radiales de las provincias, es decir, del interior del país, a diferencia del Martín Fierro principal, iniciado en 1959, destinado exclusivamente a producciones realizadas en Capital Federal. ${ }^{5}$ Entendido como una acumulación de capital simbólico (Bourdieu, 2002) el estatus social de Tarragó Ros habilita su posición como agente generador de consenso y/o disenso, lugar desde el cual postula el presente repertorio de Chamameceros exóticos.

5 Sobre la cronología del premio puede consultarse la página oficial de APTRA: https://www.aptra.org.ar/index.php/institucional/historia-de-aptra 


\section{El ChAMAMÉ Como Representativo de La Región CULTURAL DEL NORDESTE}

En la dinámica del folklore en Argentina (Díaz, 2009; Kaliman, 2004, 2006; Chamosa, 20I4) el Chamamé ha tenido un recorrido signado por una "doble marginalidad»: primero, por representar a una clase social subalterna; segundo, por no vincularse ideológica ni estéticamente con los movimientos artísticos populares legitimados al momento de su propagación metropolitana, entre 1930-1940. Según Pérez Bugallo (1996:16), «no era una expresión tradicionalista ni nativista. Es más, ni siquiera era folklore para los animadores radiales. Era simplemente chamamé. Era la música del pueblo provinciano». Pérez Bugallo (1996:4) remonta como antecedente de esta marginalidad a las prácticas musicales de la «corriente nativista» impulsada por músicos llegados a Buenos Aires desde Paraguay y Corrientes, entre 1920-1935, conformando la aparición de la denominada "música guaraní» en la metrópoli. ${ }^{6}$ En este sentido, el Chamamé fue recursivamente afiliado a la "región cultural» (Kaliman, 1999) del nordeste argentino - Corrientes, Chaco, Formosa y Misiones - en el contexto del campo artístico y cultural del folklore nacional, aunque su alcance macrorregional se extiende a Brasil, Paraguay y Uruguay. Esta representatividad regional ubica al Chamamé en el panorama de las discursividades del nacionalismo cultural (Díaz, 2009). Su reciente reconocimiento como Patrimonio Cultural Inmaterial de la Humanidad (UNESCO, 2020) testimonia un decantado proceso sociohistórico de legitimación. ${ }^{7}$

En palabras de Tarragó Ros:

Los grandes personajes heroicos del Chamamé son el pobrerío, el chamamecero que guardó el Chamamé en su corazón, cuando en su familia era considerado una lacra porque «mirá la música de mierda esta que escucha». Ese tipo resulta que pasan los ańos y esa música de mierda

6 Estos serían, según Pérez Bugallo (1996), antecesores de los pioneros de Chamamé (Cocomarola, Tarragó Ros, Montiel, Millán Medina, Abitbol).

7 UNESCO (2020) Lista Representativa de los Bienes Culturales Inmateriales, Proyecto de Decisión

15.COM 8.b.15. Disponible en: https://ich.unesco.org/en/Decisions/15.COM/8.b.15 
que escuchaba es considerada una herramienta de autoconocimiento, en el país de Flaubert, por la UNESCO ¡Y dicen que es la mejor música de toda la Argentina! (Tarragó Ros, 202I)

\section{LAS REPRESENTACIONES SOCIALES ACERCA DE LOS «CHAMAMECEROS EXÓTICOS»}

Dado que los paradigmas estéticos están constituidos principalmente por «juicios de valor» (García, 20ıо) cabe analizarlos a través de su materialidad discursiva. A su vez, siguiendo a De Nora (20I4:4):

si bien la música puede estar, parece estar o está interrelacionada con asuntos «sociales» — patrones de cognición, estilos de acción, ideologías, arreglos institucionales— esto no debe suponerse [...] más bien, es necesario demostrar sus mecanismos de funcionamiento.

Siguiendo a Moscovici (1985) y Jodelet (2002) las «representaciones sociales» son aquellos «marcos colectivos de percepción» que, a su vez, «constituyen el vínculo entre el sistema social y el sistema cognitivo individual, y además proceden a la traducción, a la homogeneización y a la coordinación de las exigencias externas con la experiencia subjetiva» (Wodak y Meyer, 2003:5I). ${ }^{8}$ Las representaciones sociales son compartidas por un grupo social pero no abarcan a todo el conjunto de la sociedad, son constructos dinámicos y permanentemente cambiantes que, como tales, constituyen un elemento nuclear de la identidad social de los individuos (Wagner, 1994:132).

8 El concepto es adoptado por Denise Jodelet (2002) a partir de la obra de Serge Moscovici (1985) en línea de continuidad con el concepto original planteado por Durkheim. Según Wodak y Meyer (2003) «en la primera mitad del siglo XIX, Émile Durkheim (1933) por ejemplo, señalaba el significado de las ideas colectivas que ayudan a las sociedades a tomar conciencia y a reificar las normas sociales" (p. 43). Tal como afirman Wodak y Meyer (2013) «Moscovici (1981) acuñó la noción de representaciones sociales entendida como la masa de conceptos, opiniones, actitudes, valoraciones, imágenes y explicaciones que es producto de la vida cotidiana y se encuentra sustentada por la comunicación" (p. 45) 
Esta perspectiva teórica concibe a los sujetos sociales en una interfaz entre lo individual/social y entre discurso/sociedad (Wodak \& Meyer 1999), y sostiene que «un individuo es inconcebible como una entidad viable sin una red sustentable de relaciones sociales» (Duveen \& Lloyd, $\left.2013^{\mathrm{a}}\right)$. En ese sentido, los discursos - tanto como los individuos- se encuentran siempre atravesados por el entramado social de una comunidad. Las representaciones sociales conforman un «sistema de valores, ideas y prácticas relacionados con un objeto específico, mientras que también se refieren al proceso a través del cual se forman tales representaciones» (Duveen \& Lloyd, 20I3b). ${ }^{9}$

En síntesis, las representaciones sociales: a) son una interfaz que mediatiza lo individual y lo social, así como el discurso y la sociedad; b) son marcos colectivos de percepción; c) son vehículos de la experiencia subjetiva; d) son sistemas de valores, ideas y prácticas; y e) son marcadores significantes de la identidad social, y de las posiciones de los individuos en la sociedad.

\section{5. «LOS CHAMAMECEROS»}

«Soy Antonio Tarragó Ros, somos Los Chamameceros» es el eslogan del ciclo radial conducido por Tarragó Ros. Este eslogan marca, junto con la cortina musical Luna payesera del propio Tarragó, la apertura de cada nuevo programa. Este eslogan define una primera relación de oposición insider/outsider de este campo cultural y artístico (Merton, 1968).

En el programa que dio comienzo al ciclo Los Chamameceros, Tarragó Ros enuncia el tópico central de dicha propuesta, de la siguiente manera: ${ }^{10}$

La alta pretensión de este encuentro con los chamameceros es ir a fondo y tratar de comprender su alma, su esencia, su pronunciamiento,

9 En su dimensión semiótica, la representación social es equiparable a un "signo" por su carácter de ser producida por/para alguien con un objetivo comunicativo, dado que, el fin (de los grupos o individuos) es «fijar su posición en relación con situaciones, acontecimientos, objetos y comunicaciones que les conciernen" (Jodelet, 1985:473).

10 En términos de Verón (1998), este enunciado inaugural instalaría un «discurso fundacional». 
su autodeterminación cultural. Y hay algunos que realmente son un abismo de hondura, de vuelo. (Los Chamameceros $\mathrm{N}^{\circ} \mathrm{I}$ )

En la novena emisión llegan los Exóticos que Tarragó Ros define como:

Un programa raro que podría llamarse "Chamameceros Exóticos». $\mathrm{O}$ sea, chamameceros que no lo son habitualmente. Y que es lindo escuchar el chamamé cuando pasa por el corazón, el sentimiento, la propuesta de alguien que no es habitualmente chamamecero. (Los Chamameceros $\mathrm{N}^{\circ}$ 9: Exóticos $\mathrm{N}^{\circ} \mathrm{I}$ )

Este «ciclo especial» se inauguró de manera casi emergente:

Tuvimos mucho éxito con el "Chamameceros Exóticos», la vez anterior; yo tenía un poco de miedo porque a veces uno escucha la radio, un programa de chamamé que uno viene siguiendo y escucha sonidos que no son muy del chamamé: orquestas, y medio se quiere ir, o que le suena más a zamba, a chacarera. Y bueno, como triunfamos, vamos a atropellarle otra vez, vamos a hacer un Número Dos, así que no se vaya, que somos los chamameceros. (Los Chamameceros $\mathrm{N}^{\circ}$ 24: Exóticos $\mathrm{N}^{\circ}$ 2)

Como puede constatarse, emergen los rasgos y características atribuidos por Tarragó Ros a los chamameceros exóticos: «Un programa de "Chamameceros Exóticos", o sea, chamameceros que cantan chamamé accidentalmente, de vez en cuando, que son exóticos, que son fuera del género, y que cantan chamamé y que son un gran aporte sin duda» (Los Chamameceros $\mathrm{N}^{\circ}$ 24: Exóticos $\mathrm{N}^{\circ}$ 2).

\section{Paradigmas estéticos}

Según Miguel A. García (20IO), «un paradigma estético puede ser concebido como un conjunto de preceptos, ordenados en una escala progre- 
siva de juicios de valor, que se desplazan desde estimaciones positivas a otras de carácter negativo»; sostiene que "es a partir de la aprobación e internalización de estos juicios estandarizados que aceptamos, rechazamos y vivenciamos las músicas que llegan a nuestros oídos» (Loc. cit.). ${ }^{11}$ Siguiendo este planteo, propongo la siguiente clasificación de atributos, en base al entramado discursivo de Tarragó Ros:

Chamamé exótico: Raro. Intérpretes accidentales (de chamamé). Cantan esporádicamente chamamé. Sonidos no-chamamé (zamba, chacarera). Orquestas. Fuera del género. Miran el chamamé con otros ojos. Otra manera de encararlo. Otros acentos. Acento exagerado (voz). Cantan otro tipo de ritmos folklóricos. Otras formas folklóricas. Otro tipo de música. Le ponen una impronta diferente.

Chamamé Canónico: Normal. Intérpretes habituales. Cantan habitualmente. Sonidos-chamamé. Solo intérpretes Chamamé. EnsamblesChamamé. Dentro del género. Autodeterminación cultural. Abismo de hondura, de vuelo. Acentos propios. Acentos adecuados. Cantan Chamamé. Admite cualquier aporte y sigue siendo Chamamé. Una música tan amplia. Música tan personal, tan fuerte.

Nos proponemos confrontar aquí estos juicios de valor con las características de las sonoridades de aquellos fonogramas incluidos entre los Exóticos. Para ello, asumiremos la perspectiva etnográfica de la audición participante (Mendívil, 20I2) como puesta en valor de la audición personal «en la cual convergen diversas y hasta antagónicas instrucciones de audición con cuestiones afectivas e ideológicas» (García, 20IO:23). Cabe tener en cuenta la premisa, sostenida por García (20I0), respecto de que «es la dimensión cultural y social de nuestros oídos aquello que condiciona en mayor medida los significados que atribuimos a los sonidos que llegan a nosotros» (Loc. cit.).

En este caso, brindaremos referencias vinculadas con los aspectos técnicos e instrumentales que pueden asumirse como «denotados»

11 Por su parte, Hennion (2010) hace referencia, antes que al concepto de juicios de valor, a la noción de "gustos" como un "pasado sedimentado (familiar, escolar, social...), es lo que forma tu identidad" (p. 28). Este autor argumenta que "no se puede continuar alternando indefinidamente entre las interpretaciones lineales-naturales (el gusto surge de las cosas en sí mismas) y las interpretaciones circulares-culturales (los objetos son lo que nosotros hacemos de ellos)" (Loc. cit.). 
en las sonoridades de los fonogramas correspondientes a los Exóticos. Asimismo, también referiremos aspectos interpretativos (y/o de la organización del material musical) que hayan sido mencionados en el entramado discursivo de Tarragó Ros (o bien, que hayan podido ser recuperados a partir de los guiones radiales del programa). No pretende ser una categorización exhaustiva, sino más bien una muestra representativa de aquellos «elementos» extrínsecos al Chamamé canónico (Tabla I).

Tabla I: Atributos de Chamamé/Chamameceros Exótico/s en oposición a Características técnicas y/o instrumentales, interpretación y organización musical).

\begin{tabular}{|c|c|c|}
\hline $\begin{array}{l}\text { Atributos del/los Chamamé/ } \\
\text { Chamameceros Exótico/s }\end{array}$ & $\begin{array}{l}\text { Características técnicas y/o } \\
\text { instrumentales }\end{array}$ & $\begin{array}{l}\text { Características } \\
\text { de la interpretaciones } \\
\text { y/o de la organización } \\
\text { del material musical }\end{array}$ \\
\hline $\begin{array}{l}\text { - Raro } \\
\text { - Intérpretes accidentales (de } \\
\text { chamamé) } \\
\text { - Cantan accidentalmente } \\
\text { (chamamé) } \\
\text { - Sonidos no-chamamé } \\
\text { - Zamba, Chacarera } \\
\text { - Orquestas } \\
\text { - Fuera del género } \\
\text { - Mirar el chamamé con otros ojos } \\
\text { (advertir cómo nos miran) } \\
\text { - Otra manera de encararlo } \\
\text { - Otros acentos } \\
\text { - Acento exagerado (voz) } \\
\text { - Cantan otro tipo de ritmos } \\
\text { folklóricos } \\
\text { - Otras formas folklóricas } \\
\text { - Otro tipo de música } \\
\text { - Le ponen una impronta diferente }\end{array}$ & $\begin{array}{l}\text { - Conjuntos o ensambles «vocales» } \\
\text { (con o sin instrumentación) } \\
\text { - Aerófonos andinos } \\
\text { - Armónicas } \\
\text { - Percusión latina: congas, } \\
\text { timbales, maracas. } \\
\text { - Címbalos } \\
\text { - Drum-machine } \\
\text { - Guitarra acústica, Guitarra } \\
\text { Ovation } \\
\text { - Guitar Slides } \\
\text { - Piano acústico, piano eléctrico } \\
\text { - Bajo eléctrico, contrabajo } \\
\text { - Drum-Machine } \\
\text { - Sintetizadores: strings, samples } \\
\text { de guitarras y/o acordeones } \\
\text { - Batería electrónica } \\
\text { - Efectos: Reverberancia, Chorus, } \\
\text { Delay. }\end{array}$ & $\begin{array}{l}\text { - Voces: extensiones vocales } \\
\text { amplias, articulaciones legato, } \\
\text { vibratos, enmascaramiento, } \\
\text { dinámicas pianíssimo, fortíssimo, } \\
\text { estéticas belcanto. } \\
\text { - Arreglos: conducción de } \\
\text { voces, armonías, contrapuntos. } \\
\text { Solos. Armonía con dominantes } \\
\text { secundarios, acordes con } \\
\text { tensiones } 9^{a} / 11^{\mathrm{a}} \text {. } \\
\text { Timbre de las voces, los } \\
\text { acordeones, las guitarras. } \\
\text { Textos en hebreo } \\
\text { Presencia de otros géneros: } \\
\text { Chamarrita, Rasguido Doble. } \\
\text { - Acción performativa de los } \\
\text { intérpretes y/o productores, } \\
\text { arregladores: Alberto Cortéz, } \\
\text { Bebu Silvetti (Luis Miguel), Ricard } \\
\text { Miralles (Joan Manuel Serrat), } \\
\text { León Gieco, Miguel Cantilo, } \\
\text { Bernhard Von Der Goltz, Mercedes } \\
\text { Sosa, Chalchaleros. }\end{array}$ \\
\hline
\end{tabular}

\section{Exotismo y AUtoexotismo}

Siguiendo a Harootunian (2012:25) la corriente de pensamiento que reflexiona críticamente sobre el exotismo fue iniciada por Victor Segalen (I878-1919) a través de su Ensayo sobre exotismo (publicado en 1955) el cual es considerado como un importante antecedente en la discusión 
sobre alteridad cultural y poscolonialismo en el debate socioantropológico contemporáneo (Ibid. 25). ${ }^{12}$

Interpretamos aquí el exotismo propuesto por Tarragó Ros no como una reificación de la alteridad, ni como una negación de lo diferente o diverso - lo cual es más propio de las perspectivas que consideran al exotismo desde enfoques reduccionistas (García 2009:43). Al contrario, en la complejidad del tipo de exotismo que analizamos, entendemos que se trata de un realce positivo de la diferencia, cuyo destinatario no deja de ser un auditorio radiofónico al cual Tarragó Ros buscó acercar una nueva sonoridad a través de la diferencia: «yo quería hacerles escuchar a los chamameceros — que eran mi público casi exclusivamenteestos sonidos del chamamé cantados por otra gente que no era del palo» (Tarragó Ros, 202I).

El término autoexotismo pertenece a Izquierdo-König (20I6) quien lo aplica en el campo de la musicología latinoamericana, definiéndolo en dicho contexto como:

La creación de una América Latina exótica como producto académico dentro de un mercado que promueve tal diferenciación como marca distintiva de presencia, en detrimento de una búsqueda de posibilidades de profundización de un aporte historiográfico -y musicológico al fin- construido desde América Latina en un diálogo. (IzquierdoKönig, 20I6:96)

En nuestro caso en particular, el autoexotismo — aplicado al campo cultural y artístico de las prácticas musicales del Chamamé por un insider de la cultura chamamecera- se configura a través de un laborioso proceso (la emisión y recepción de todo el ciclo Los Chamameceros) mediante el cual —en un entramado sociodiscursivo y en un agrupa-

12 Según Ludeña Aranda (2016), Víctor Segalen no solo sobresale por haber sido «el primer autor que se plantea escribir un ensayo sobre el exotismo [...] en 1904; tarea que abordará hasta 1918 en distintos escritos que configuran un ensayo fragmentario" (Loc. cit.) sino que también fue creador del término "exota" (Ibid.:13). La corriente ensayística del exotismo continuaría en escritos de autores como: Lily Litvak (exotismo y erotismo), Tzvetan Todorov, Gabriel Weisz y el propio Edward Said —con su categoría de "Oriente» como invención de Occidente (Ludeña Aranda, 2016). 
miento selectivo de obras musicales - Tarragó Ros configuró el paradigma estético de los Chamameceros Exóticos resaltando, por oposición, el paradigma estético del Chamamé Canónico.

\section{Consideraciones finales}

En el presente trabajo, la caracterización de los paradigmas estéticos Exótico/Canónico (sus rasgos sonoros y los juicios de valor atribuidos a los mismos) mediante el análisis de las representaciones sociales nos permitió visibilizar, por una parte, la posición asumida por Tarragó Ros como representante legítimo del género- en la dinámica de inclusión/ exclusión en dichos paradigmas. Por otra parte, también hemos visibilizado la forma en que un músico vernáculo concibe y asume sus propias prácticas musicales y el modo en que estas se inscriben en los paradigmas estéticos de referencia. Cabe destacar que Tarragó Ros también se incluye a sí mismo dentro de estos Chamameceros Exóticos en aquellas obras musicales en las que interviene y participa como intérprete, arreglador o productor. Este rasgo de su autoexotismo performa una vocación de diálogo entre géneros musicales, a través de las prácticas musicales de estos artistas y una capacidad de negociación simbólica dentro de diversas configuraciones culturales que acogieron al Chamamé como medio válido de expresión artística a lo largo de este ciclo radiofónico.

En este sentido, los géneros musicales «continúan siendo categorías operativas de creación artística y de uso analítico» (Ochoa, 2003:84); sin embargo, asumimos que no se trata de una categoría homogénea. Según Fabbri (20I4:32), «todos los conceptos taxonómicos musicales juegan un papel central en la creación musical [...] en la teoría y en la crítica musical, como en cualquier tipo de comunicación verbal sobre música». Siguiendo a Drott (20II:85), «cualquier valor o significado que adquieran los géneros individuales es producto de su relación con otros géneros y de la posición que ocupan dentro de todo el sistema de géneros». Por ello, la definición de los géneros, lejos de ser estática, resulta de «actos de ensamblaje realizados por agentes específicos en contextos 
sociales e institucionales específicos» (Ibid. Io). Es decir, además de las prácticas musicales y sus rasgos formales - que diferencian una construcción musical de otra- los géneros también son construidos ideológicamente por los agentes que buscan homogeneizar unos rasgos por sobre otros, borrándose, en última instancia, los rasgos no-deseables (Ochoa, 2003:87).

En el presente caso, el autoexotismo musical aquí analizado se constituye como parte del ensamblaje simbólico del Chamamé. Esta dinámica de inclusión/exclusión no solo alcanza a las prácticas musicales o a los géneros musicales en una dimensión meramente formal sino que, a su vez, manifiesta una gran capacidad de incidencia en la conformación de todo un campo cultural, intelectual y artístico (como lo es la música popular argentina) atravesado por tensiones, luchas por la legitimidad y el poder simbólico. Esto nos permite interpretar que un género musical - entendido como categoría formal, como conjugación de paradigmas estéticos- no representa nada si no está vinculado con la comunidad que le da sentido.

El autoexotismo aquí analizado no expresa un ensamblaje de tipo «endogámico» que busca «clausurar» al Chamamé frente a la alteridad; al contrario, el repertorio de Exóticos muestra que el lugar protagónico lo tienen esas "otras voces, otros sonidos, otras improntas», que refuerzan la legitimidad simbólica de un género que «admite cualquier aporte y sigue siendo Chamamé» (Tarragó Ros, 202I). En este caso, esas alteridades son artistas de gran reconocimiento y trayectoria en otros géneros, procedentes desde dentro y fuera del país.

Esto nos permite concluir que todas estas prácticas y producciones musicales no hacen más que enfatizar el devenir de un género de origen subalterno y marginal, que fue ganando espacios de aceptación y legitimación, durante varias décadas, hasta obtener el reciente reconocimiento como Patrimonio Cultural Inmaterial de la Humanidad. 


\section{REFERENCIAS BIBLIOGRÁFICAS}

ABRIC, JEAN Claude (1994). Prácticas sociales y representaciones, México: Ediciones Coyoacán.

Bourdieu, Pierre (2002). Campo de Poder, Campo Intelectual. Itinerario de un concepto, Montressor.

CUVARDIC GARCÍA, DORDE (2012). El flâneur en las prácticas culturales, el costumbrismo y el modernismo. Sciences Humaines et sociales. Collection Terres Hispaniques dirigée par Nicolas Balutet. Publibook Université.

DE NORA, TIA (2004). Música en la vida cotidiana. Cambridge University Press.

DÍAZ, CLAUdio (2009). Variaciones sobre el "ser nacional»: una aproximación sociodiscursiva al folklore argentino, Córdoba: Ediciones Recovecos.

DROTT, ERIC (2011). Music and the elusive revolution: Cultural politics and political culture in France, 1968-1981, University of California Press.

DUVEEN, GERARD \& LLOYD, BARBARA (2013a). The significance of social identities. En MOSCOVICI, S., JOVCHELOVITCH, S. y WAGONER, B. (Eds.) Development as a social process: Contributions of Gerard Duveen. Routledge. DUVEEN, GERARD \& LLOYD, BARBARA (2013b). Social representations as a genetic theory. En MOSCOVICI, S., JOVCHELOVITCH, S. y WAGONER, B. (Eds.) Development as a social process: Contributions of Gerard Duveen. Routledge. FABBRI, FRANCO (2014). Music taxonomies. Conference Musique Savante/ Musiques Actuelles: Articulations. JAM: Journées d'analyse musicale, Société Française d'Analyse Musicale, Paris.

GARCíA, Miguel A. (Ed.) (2010): Rock en papel: Bibliografía crítica de la producción académica sobre el rock en Argentina. Editorial de la Universidad de La Plata.

GARCÍA, MIGUEL A. (2009). Literaturas postcoloniales, hibridación y exotismo, Prosopopeya. Revista de Crítica contemporánea, 6, 41-66.

HAROOTUNIAN, HARRY Y SEGALEN, VICTOR (2002): Essay on exoticism: an aesthetics of diversity. Stanley Fish and Fredric Jameson (Series Ed.). Duke University Press.

HENNION, ANTOINE (2010). Gustos musicales: de una sociología de la mediación a una pragmática del gusto, Comunicar, 17(34), 25-33.

IZQUIERDO KÖNIG, JOSÉ MANUEL (2016). Auto-exotismos: la musicología latinoamericana y el problema de la relevancia historiográfica (con un apéndice sobre música sacra y el siglo XIX), Resonancias, 20(38), 95-116.

JODELET, DENIS (1993). La representación social: fenómenos, concepto y teoría. En Moscovici, S. (Ed.) Psicología Social II, Paidós.

KALIMAN, RICARDO (2006). Criollos y criollismo en la industria del folclore musical, Revista de Investigaciones Folklóricas, 21, pp. 88-93.

KALIMAN, RICARDo (2004). Alhajita es tu canto El capital simbólico de Atahualpa Yupanqui, Córdoba: Comunicarte. 
KALIMAN, RICARDO (1999). Un marco (no 'global') para el estudio de las regiones culturales, Journal of Iberian and Latin American Research, 5(2), pp. 11-22. DOI: 10.1080/13260219.1999.10431795

LUDEÑA ARANDA, LUCíA (2016). El grupo Shangai y el exotismo en la novela argentina de las dos últimas décadas del siglo XX, Doctoral dissertation, Universidad Complutense de Madrid.

MENDÍvil, JULIO (2012). "Dos, tres, grabando": la tecnología del sonido y naturalización de los medios en el caso del huayno peruano, Memorias del II Encuentro Internacional de Musicología. Vol. 1, Musicología desde Ecuador, 22 a 26 de octubre.

MERTON, ROBERT KING (1968). Social Theory and Social Structure, New York: The Free Press.

MERRIAM, ALAN (1979). The anthropology of music, Northwestern University Press. MOSCOVICI, SERGE (1979). El Psicoanálisis, su imagen y su público, Buenos Aires: Huemul.

OCHOA, ANA MARÍA (2003). Músicas locales en tiempos de globalización, Buenos Aires: Norma.

PÉREZ BUGALLO, RUBÉN (2005). Tradicionalismo, nativismo y proyección folklórica en la música argentina. Labor y huella de los Gómez Carrillo, Estudios y documentos referentes a Manuel Gómez Carrillo, San Isidro, Academia de Ciencias y Artes de San Isidro. Disponible en:

HTTP://WwW.FUNDACIONCULTURAL.ORG/REVISTA/NOTA6_I 2.HTML

SEGALEN, víctor (2017). Ensayo sobre el exotismo: una estética de lo diverso. Martín Schifino (trad.), Cuadernos de horizonte, Serie Azimut, España, La línea del Horizonte.

SEGALEN, víctor (2002). Essay on exoticism: an aesthetics of diversity. Stanley Fish and Fredric Jameson (Series Ed.). Duke University Press.

SUPIČIČ, IVo (1988). Sociología musical e historia social de la música. Neus Arqués, (Trad.) en Papers: revista de sociologia, pp. 79-108. Título original: "Perspectives pluridisciplinaires: difficultés d'approche" (1985), enc International Review of the Aesthetics and Sociology of Music, 16 (1985) 2, pp. 125-151.

SUPIČIČ, IVO (1987). Music in society: A guide to the sociology of music. Pendragon Press.

TARRAGó ROS, ANTONIO (2021). Entrevistas realizadas durante 20-04-2021 y 30-04-2021 por Emanuel Obregón. Archivo personal.

THIBAUDEAU, PASCALE (2007). Del repertorio musical y coreográfico al repertorio cinematográfico: el caso de Carlos Saura, Pandora: revue d'etudes hispaniques, (7), 21-30.

VERÓN, ELISEO (1998). La semiosis social: fragmentos de una teoría de la discursividad, Madrid: Gedisa.

WODAK, RUTH Y MEYER, MICHAEL (1999). Métodos de análisis crítico del discurso, Barcelona: Gedisa. 